\title{
IMPROVED INDOOR LOCALIZATION WITH DIVERSITY AND FILTERING BASED ON RECEIVED SIGNAL STRENGTH MEASUREMENTS
}

\author{
Andreas Fink, Helmut Beikirch, Matthias Voss \\ University of Rostock, Department of Computer Science and Electrical Engineering \\ A.-Einstein-Str. 2, D-18059 Rostock, Germany \\ \{andreas.fink;helmut.beikirch;matthias.voss2\}@uni-rostock.de \\ www.e-technik1.uni-rostock.de
}

\begin{abstract}
Distance estimation by the evaluation of RSSI measurements is a simple and well-known technique to predict the position of an unknown node. Therefore the infrastructure does not have to be extended by expensive hardware for synchronization or direction approximation. However, with the localization based on RSSI measurements common and proven systems can be used for the infrastructure. For indoor environments the distance-pending path loss is affected by strong variations, especially appearing as frequency specific signal dropouts. A diversity concept with redundant data transmission in different frequency bands can reduce the dropout probability. If also space diversity and plausibility filtering are used, the Location Estimation Error can be reduced significantly. The investigations show that a good performance for precision and availability can also be reached with low infrastructural costs.
\end{abstract}

Keywords: Diversity Combining, Indoor Localization, Plausibility Filter, RSSI Reading.

\section{INTRODUCTION}

The operation of safety-relevant wireless communications in automation-technical applications is to become more reliable by the monitoring of mobile network participants with a sufficiently exact estimation of their position [1]. That means for instance, it is possible to recognize that mobile network participants are leaving a sensitive area on time. Thus not necessarily the precise position but a rough movement pattern is of interest. The positioning in this surrounding field takes place primary within indoor environments (factory buildings and manufacturing facilities) which do not permit a positioning with GPS (or only with poor quality). For that reason the actual data communication between the mobile network participants seems better suitable for the positioning in this environment. RSSI (Received Signal Strength Indicator) measurements for the estimation of distances between transmitter and receiver are a well-known technique to predict the position of an unknown node [2]. Therefore the infrastructure do not have to be extended by expensive hardware for synchronization (like for Time of Arrival or Time Difference of Arrival measurements) or direction approximation (like for Angle of Arrival measurements).

\section{SYSTEM REQUIREMENTS}

Especially for indoor environments the distancepending path loss is affected by strong variations (NLOS conditions, signal fading). Frequency specific signal dropouts in case of destructive interference effects as a result of the multipath propagation lead to bad distance approximations (cf. Fig. 1).

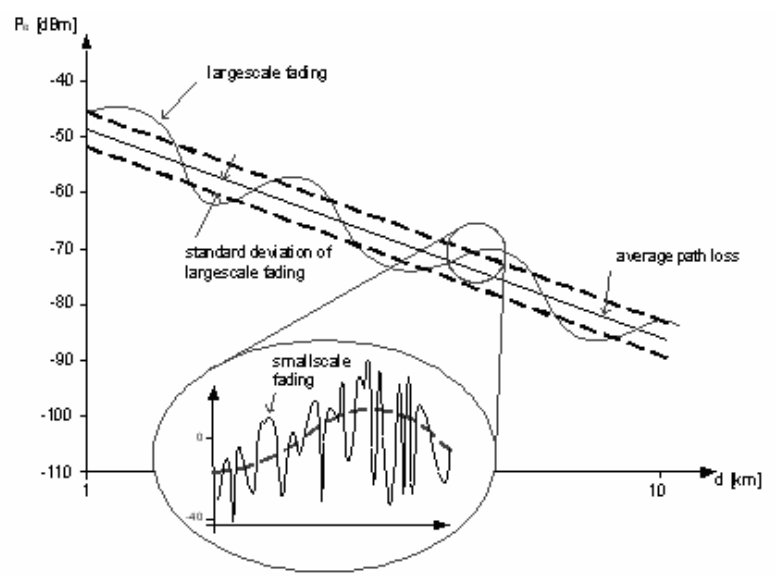

Fig. 1 - Distance-depending path loss affected by fading [3]

When designing an RSSI-based location-sensing system it is recommended to concentrate on the proper acquisition of the RSSI values since their 
interpretation has the main influence on the system's accuracy. Therefore we use a diversity concept (cf. Fig. 2) to reduce the dropout probability. As described in [4] and [5], several statistically independent channels are formed with diversity which makes a combining of the received signals possible by the redundant data transmission.

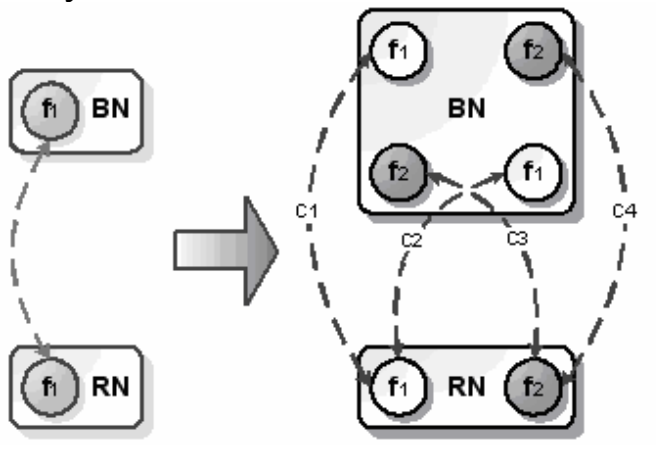

Fig. 2 - Diversity concept for a reduced dropout probability

The unknown node (Blind Node, $B N$ ) is equipped with four RF modules which are arranged in a rectangle (from the top view). Further two different frequency bands (868 $\mathrm{MHz}$ and $2.4 \mathrm{GHz}$ ISM radio) are used, whereby the diagonal arranged modules use the same frequency band. Beside space diversity also frequency diversity is realized for both transmission directions. The exemplary illustrated Reference Node (RN) has two RF modules which use different frequency bands. Between $\mathrm{BN}$ and $\mathrm{RN}$ altogether four uncorrelated channels (C1..C4) are formed. In comparison only one channel is formed with the conventional communication scheme. How the number of channels is linked up to the availability of the wireless interface and thus also to the accuracy of the position estimation is described in [6].

A further requirement for the system is a possible influence of the electric field distribution by moving macroscopic objects in the area (e.g. mobile robots, humans). A comparison of the filtered sensor data with a predefined knowledge base [7] is therefore not possible for the position estimation. Thus optimization methods like the use of detailed RSSI maps or Ray Tracing techniques are not possible since the accuracy is reached by a static reflection of the environment with these methods.

The mobile BN can move around with a maximum speed of $5 \mathrm{~m} / \mathrm{s}$. This acceptance is important for the adjustment of the calculated distances through a dynamic filtering, whereas temporary fluctuations of the signal strength (caused by small-scale fading) can be detected and evaluated. In order to get a plausible interpretation of the occurring fading effects with the dynamic filter, a time scaling [2] of 5 values per second is defined as the minimum actualization rate of the position computation. Between two computed positions the $\mathrm{BN}$ then can travel a maximum distance of $1 \mathrm{~m}$. A lower actualization rate reduces the effectivity of the filter, whereby a higher rate is restricted by the limited capabilities of the a priori simple hardware components.

The system's main features arising out of the mentioned requirements can be summarized as follows:

- Acquisition and evaluation of the static environmental conditions

- Plausibility filtering based on the dynamics

- Diversity concept with selection combining of the redundant RSSI values

- Adapted localization algorithm (resistant in relation to strong variations of RSSI values)

\section{INFRASTRUCTURE COMPONENTS}

With the localization based on RSSI measurements common and proven systems (LowPower microcontroller, proprietary RF-transceiver) can be used for the infrastructure. The localization system has a complex distributed architecture. The infrastructure consists of eight fixed RNs, the mobile $\mathrm{BN}$ and a PC for visualization and analysis of the localization (cf. Fig. 3). Further, the RNs are connected over the CAN bus with each other and to the Analysis PC.

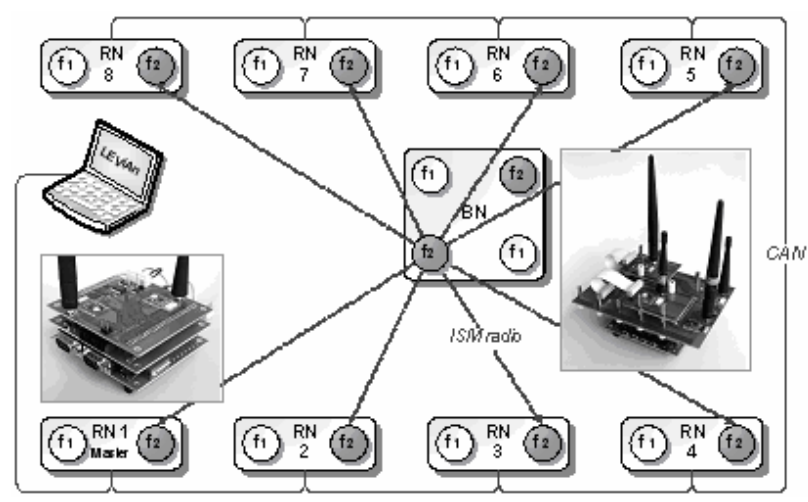

Fig. 3 - System overview with infrastructure components

The MSP430F169 Low-Power MC from TI is used as system controller on the BN (cf. Fig. 4) and on the RN (cf. Fig. 5).

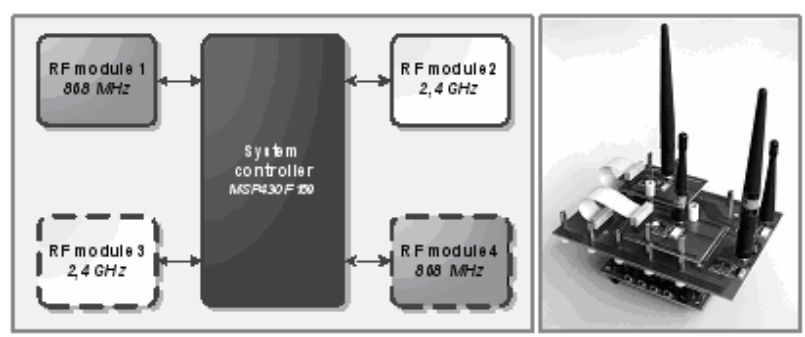

Fig. 4 - System architecture (left) and prototype (right) of the $\mathrm{BN}$ 
The RF communication takes place by proprietary radio modules from Amber Wireless. For the diversity concept the CC1100-based AMB8420 and the CC2500-based AMB2520 are used which operate at license-free ISM bands $(868 \mathrm{MHz}$ respectively $2.4 \mathrm{GHz}$ ). The CAN interface on the RN consists of the MCP2515 CAN controller from Microchip and the SN65HVD CAN transceiver from Texas Instruments. For the connection to the Analysis PC the Lawicel CAN-USB Converter is used.

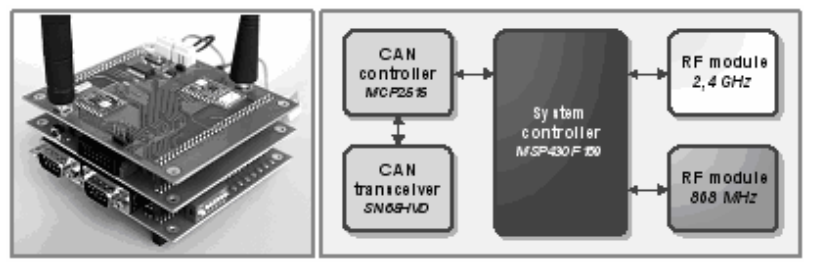

Fig. 5 - System architecture (right) and prototype (left) of a RN

Besides the firmware of the Embedded Systems a Java application (LEViAn - Location Engine With Visualization and Analysis) was developed which can be used for the evaluation and analysis of the localization on the Analysis PC. The Radio Map is a central component of the application (cf. Fig. 6) and enables a visualization for the positioning over a circular representation of the calculated distances between $\mathrm{BN}$ and RNs. In addition to the fixed RN's positions the actual and the calculated position of the $\mathrm{BN}$ are shown in this map.

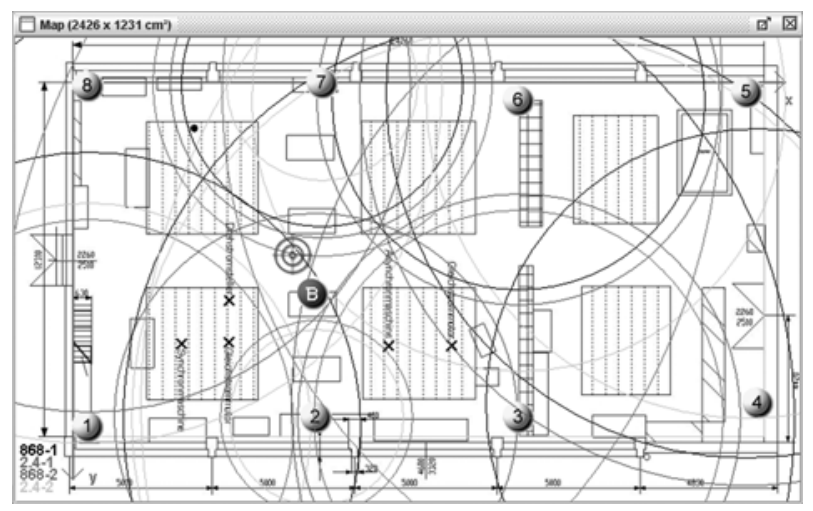

Fig. 6 - Radio Map of the LEViAn application

\section{POSITION ESTIMATION TECHNIQUES}

Different intermediate steps are provided to compute a position information based on RSSI values. The localization system mainly can be subdivided into the components data acquisition, data preprocessing and localization (cf. Fig. 7).

As mathematical model for the distancedependent path loss at the propagation of electromagnetic waves the Log Distance Model [8] according to (1) and (2) is used.

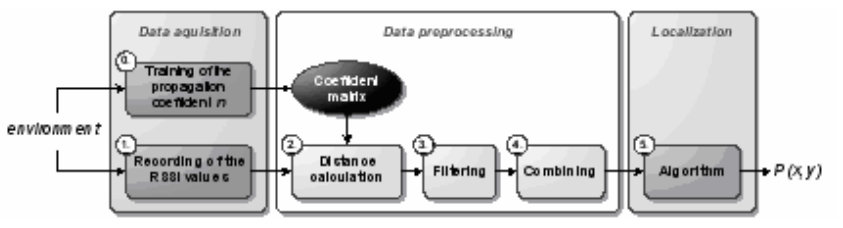

Fig. 7 - Structure of the developed localization system

Before the actual positioning the acquisition of the static environmental conditions takes place by the training of the propagation coefficient $(n)$ and the determination of the path loss offset (A) (measured in a distance of $1 \mathrm{~m}$ to transmitter) which characterizes the used path loss model. The training of $n$ is realized by a data exchange between all RNs in an initial setup phase. With the RN's fixed position and the collected RSSI values the RNspecific $n$ is given by

$$
n=\frac{R S S I_{d B m}-A}{10 \cdot \log d} .
$$

With the training an automatic optimization of the infrastructure with an adaption to the environmental conditions is possible. There is no need of manually collecting RSSI data like for many other localization systems (e.g. [7]).

The acquisition of the redundant RSSI values is made during the online phase by the periodic data transmission between $\mathrm{BN}$ and $\mathrm{RN}$. With the constraint that the $\mathrm{BN}$ have to transmit data (e.g. sensor values like temperature, acceleration etc.) periodically the acquisition of the RSSI value for the localization needs no additional communication.

According to (2) first a distance is calculated from the recorded RSSI values during the data preprocessing phase.

$$
d=10^{\left(\frac{R S S I_{d B m}-A}{10 \cdot n}\right)}
$$

Subsequently, a plausibility filtering of the distances takes place based on the dynamics. With the constraint that the $\mathrm{BN}$ can move around with a maximum speed ( $v_{\max }$ ) of $5 \mathrm{~m} / \mathrm{s}$ and the time stamps of two consecutive position requests we are able to compute a possible maximum change in distance $\left(\Delta d_{\max }\right)$ according to (3).

$$
\Delta d_{\text {max }, n}=\left(t_{n}-t_{n-1}\right) \cdot v_{\max }
$$

The output values of the dynamic filter are then computed with (4).

$d_{n}^{\prime}=\left\{\begin{array}{cl}d^{\prime}{ }_{n-1}+\Delta d_{\max , n} & ,\left(d_{n}-d^{\prime}{ }_{n-1}\right)>\Delta d_{\max , n} \\ d^{\prime}{ }_{n-1}-\Delta d_{\max , n} & ,\left(d^{\prime}{ }_{n-1}-d_{n}\right)>\Delta d_{\max , n} \\ d_{n} & , \text { else }\end{array}\right.$ 
By the used diversity concept, the corrected values are redundant. Hence, the smallest calculated distance between $\mathrm{BN}$ and the appropriate $\mathrm{RN}$ is chosen by selection combining.

The actual position estimation is made by a Weighted Centroid Localization (WCL) which has been adapted to the specific environmental conditions. With the AWCL (Adaptive Weighted Centroid Localization) a good theoretical approach is already given in [9]. When looking at real scenarios with high fluctuating RSSI values an enhancement of this algorithm is useful. The new defined SAWCL (Selective Adaptive Weighted Centroid Localization) do not only treat high LQIs ${ }^{1}$ (like AWCL) but also adapt low LQIs (cf. Fig. 8).

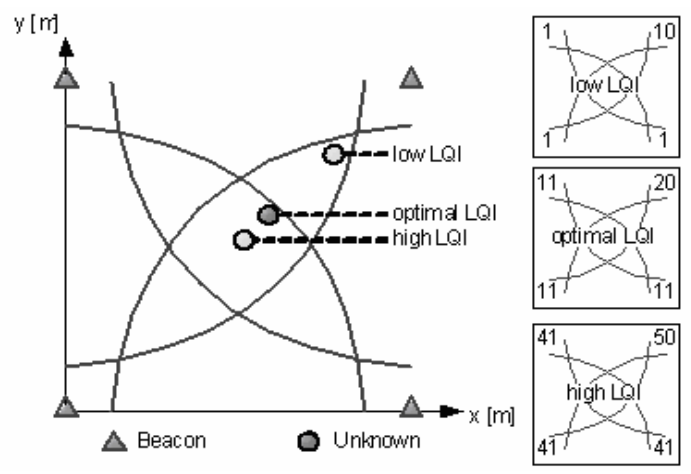

Fig. 8 - Influence of the RSSI's distribution on accuracy for WCL [9]

First a transformation of the distances into weights takes place similar to the WCL according to (5).

$$
w_{i j}=\frac{1}{\left(d_{i j}\right)^{g}}
$$

Dependent on the statistic distribution of the weights an adjustment of the values is made afterwards. The ratio $r_{b}$ is given by

$$
r_{b}=\frac{m-1}{m} \cdot \frac{\left(\sum_{j=1}^{m} w_{j}\right)}{\left(\sum_{j=1}^{m} w_{j}\right)-w_{\max }}
$$

and indicates the influence of the maximum weight compared to all other weights. The weights are all reduced or raised by a factor which is affected by the RSSI's variance. Therefore another parameter $b$ can be defined which characterizes the border for the decision whether to reduce or raise the weights. For the regarded environment a value of 1.5 for $b$ was determined. The values of $q_{r e d}$ and $q_{a d d}$ describe the corresponding factors and are given by

$$
q_{\text {red }}=\frac{b-r_{b}}{b-1} \quad \text { and } \quad q_{\text {add }}=\left(r_{b}-b\right) .
$$

\footnotetext{
${ }^{1}$ Link Quality Indicator, e.g. RSSI values
}

The adjustment technique for a set of four RNs and a normalization to the lowest weight is shown in Fig. 9.

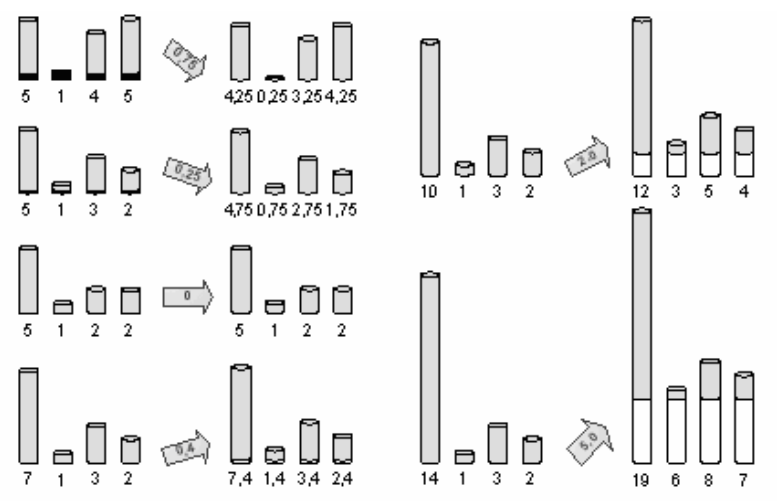

Fig. 9 - Adjustment of weights according to their statistic distribution

In the last step the BN's position $p(x, y)$ is computed with the modified weights and the fixed positions $B(x, y)$ of the RNs according to (8). A detailed description of the used techniques can be found in [4].

$$
P_{i}(x, y)= \begin{cases}\frac{\sum_{j=1}^{n}\left[\left(w_{i j}-q_{r e d} \cdot \min \left(w_{i, 1 . . n}\right)\right) \cdot B_{j}(x, y)\right]}{\sum_{j=1}^{n}\left(w_{i j}-q_{r e d} \cdot \min \left(w_{i, 1 . n}\right)\right)} & , r_{b}<b \\ \frac{\sum_{j=1}^{n}\left[\left(w_{i j}+q_{a d d} \cdot \overline{w_{i, 1 . . n}}\right) \cdot B_{j}(x, y)\right]}{\sum_{j=1}^{n}\left(w_{i j}+q_{\text {add }} \cdot \overline{w_{i, 1 . . n}}\right)} & , \text { else }\end{cases}
$$

\section{EXPERIMENTAL RESULTS}

The plant floor of the Institute of Electrical Power Engineering at the University of Rostock was chosen as reference environment for the developed system (cf. Fig. 10).

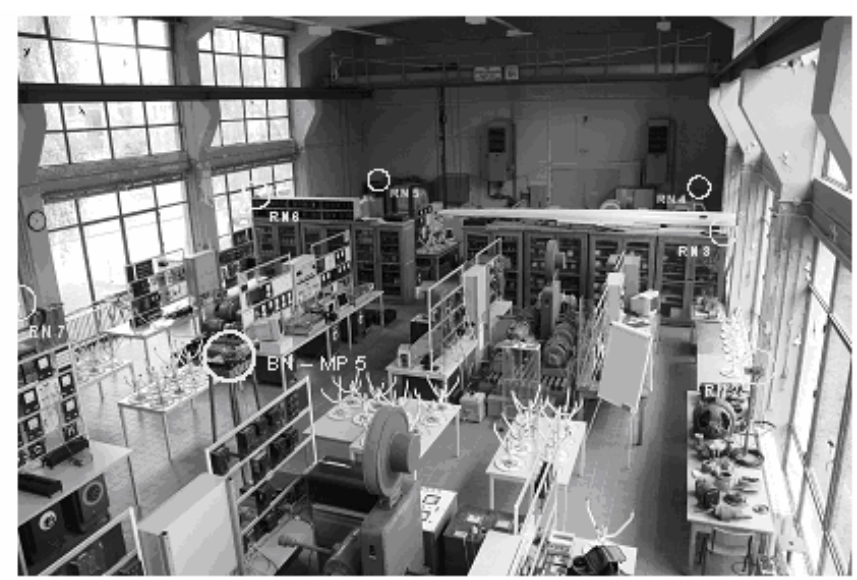

Fig. 10 - Test bed: obstructed plant, one floor, $12 \mathrm{~m} \mathrm{x}$ $24 \mathrm{~m}$

With a footprint of approximately $24 \mathrm{~m} \times 12 \mathrm{~m}$ and several larger electrical machines (direct current motor, asynchronous machine) as well as further 
macroscopic objects (shelves, switchboards) the environment is particularly suitable for the measurement under conditions which are similar to the industrial surrounding field. The eight RNs are evenly distributed at the edge of the regarded footprint at a level of $2.5 \mathrm{~m}$ above the ground. The horizontal distribution is shown in the Radio Map in Fig. 6. The positions of the RNs with ID 2 to 7 are also represented in Fig. 10.

For the test bed (cf. Fig. 10) extensive measurements were carried out. For 12 fixed measuring positions in each case 1,000 positions were calculated. A resolution of 5 values per second was selected for the system's time scaling. To avoid random adjustments of the BN's antennas the device rotates with $33 \mathrm{rpm}$ around the center between the four antennas. Thus, all possible adjustments are considered for the computation of the LEE.

The corresponding values for the LEE are shown in Tab. 1 for various configurations. The settings differ in the applied filtering technique and in the used number of communication channels between $\mathrm{BN}$ and $\mathrm{RN}$.

Table 1. Comparison of different configurations (error in meters)

\begin{tabular}{|r|c|c|c|c|c|c|}
\hline Parameter & \multicolumn{7}{|c|}{ Configuration } \\
\hline & a) & b) & c) & d) & e) & f) \\
\hline \# channels 868 MHz & 1 & 1 & & & & \\
\hline \# channels 2.4 GHz & - & - & 1 & 1 & 2 & 2 \\
\hline Plausibility filter & - & yes & - & yes & - & yes \\
\hline LEE $_{\text {av }}$ & 2.56 & 1.91 & 1.90 & 1.50 & 1.13 & 1.00 \\
\hline LEE $_{\mathbf{m e d}}$ & 1.80 & 1.30 & 1.30 & 1.30 & 1.08 & 1.02 \\
\hline$\sigma_{\mathbf{L E E}}$ & 2.10 & 1.50 & 1.58 & 1.09 & 0.75 & 0.58 \\
\hline & 6.86 & 5.69 & 5.10 & 4.21 & 2.60 & 2.05 \\
\hline & $\mathbf{9 . 0 9}$ & 6.02 & 6.44 & 4.40 & 3.45 & 2.47 \\
\hline & & & &
\end{tabular}

The results for the CDF of the LEE are represented in Fig. 11 for different configurations. Hence, the profit in accuracy through the filtering and the diversity concept is pointed out.

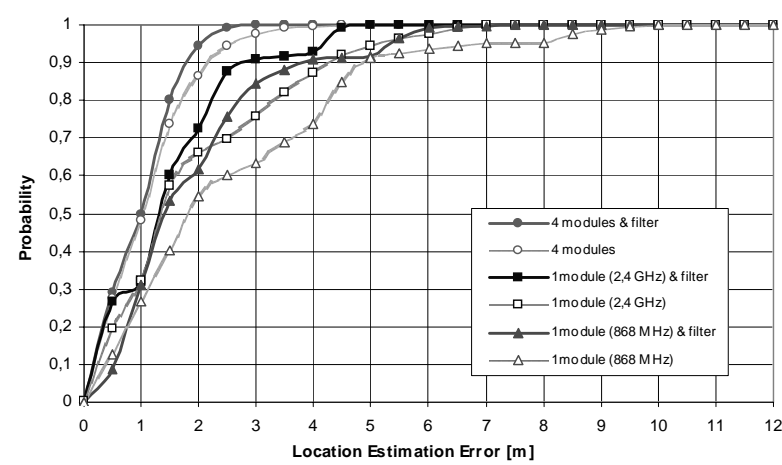

Fig. 11 - CDF - Location Estimation Error for different configurations

A LEE smaller than $2.5 \mathrm{~m}$ for the $99^{\text {th }}$ percentile is reached with the combining of four redundant
RSSI values and plausibility filtering (Configuration f). Without combining and filtering (Configuration a) the $99^{\text {th }}$ percentile of LEE is already exceeding 9 $m$ for one of the $868 \mathrm{MHz}$ channels. So the 99th percentile has been reduced by more than $72 \%$ compared to the conventional solution.

Beside the CDF also the distribution of the computed positions over the footprint of the reference environment can be used as valuation criterion for the localization system. In Fig. 12 the distribution of the BN's calculated positions at one fixed measuring position are shown as a 3D scatterplot for the configuration a) (1 module@868 $\mathrm{MHz}$, filter off). A very strong dispersion of the values can be determined. It is obvious that a localization in an obstructed plant with the mentioned fading effects is only possible with poor quality if no additional techniques are used.

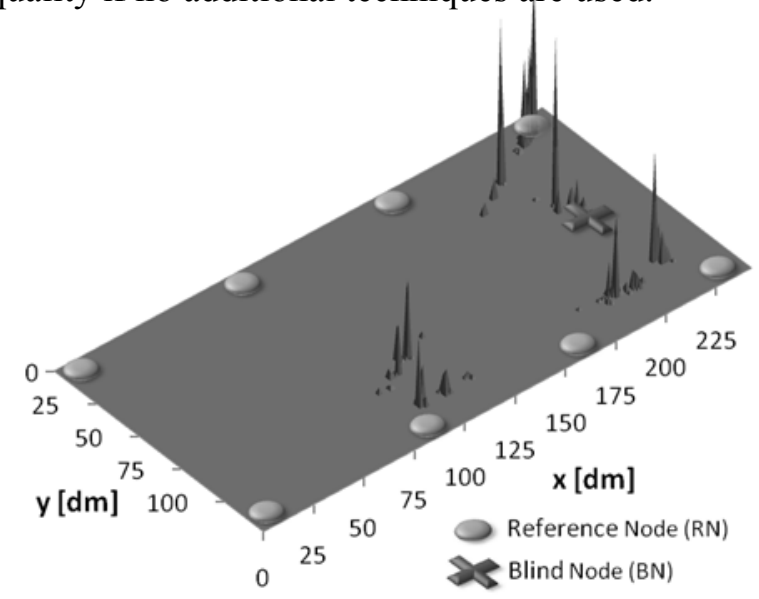

Fig. 12 - Distribution of calculated positions over the footprint of the plant - configuration a): 1 module @ $868 \mathrm{MHz}$, filter off

When a plausibility filtering is added to the processing line a higher accuracy can be achieved as represented by the distribution in Fig. 13 for configuration $b$ ). The filtering is important especially for tracking applications.

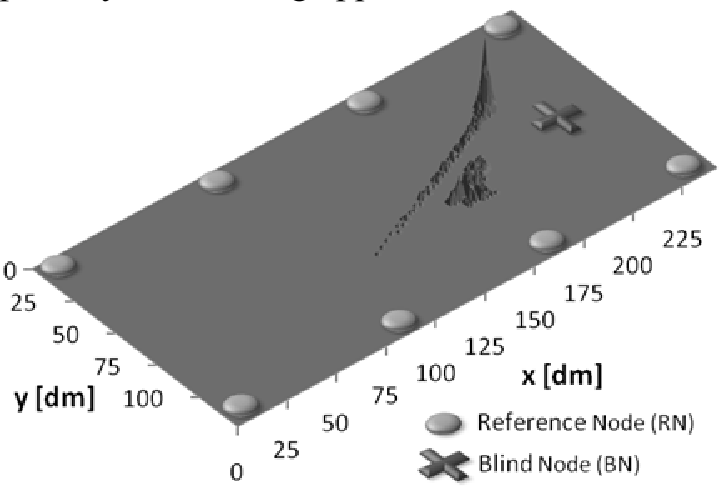

Fig. 13 - Distribution of calculated positions over the footprint of the plant - configuration b): 1 module @ $868 \mathrm{MHz}$, filter on 
Even if the Location Estimation with the filter is more accurate the distribution shows a systematic error because no coherence between the computed positions and the BN's real location can be found. This behavior can be eliminated with the used diversity concept (configuration e). In Fig. 14 the BN's calculated positions are distributed around its real location.

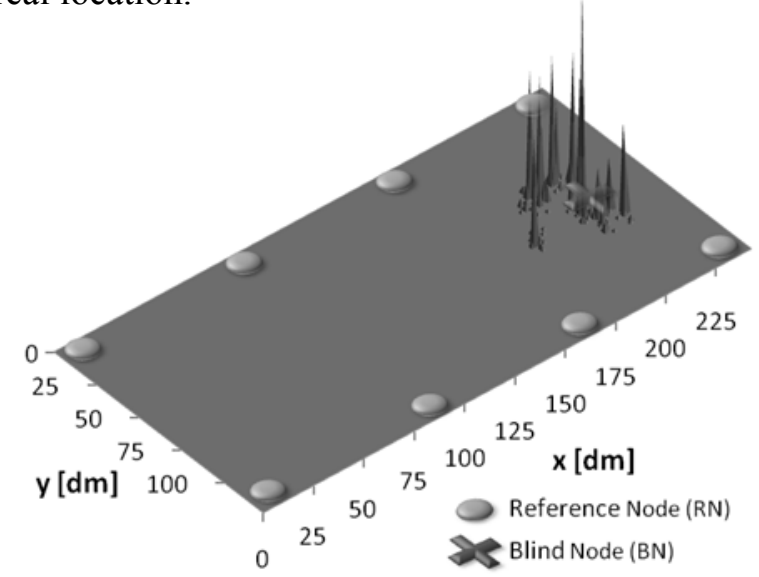

Fig. 14 - Distribution of calculated positions over the footprint of the plant - configuration e): 4 modules, filter off

As the distribution for configuration $f$ ) in Fig. 15 shows, only the combination of a diversity concept with four uncorrelated communication channels and a plausibility filtering enables a sufficient accuracy for a rough movement pattern of the BN.

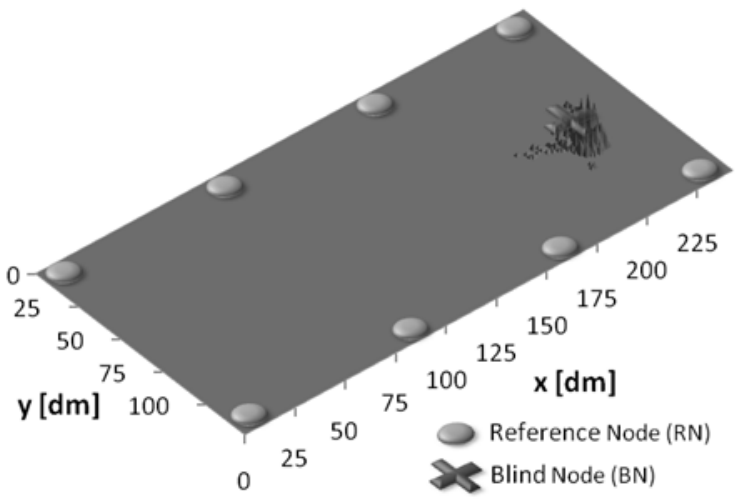

Fig. 15 - Distribution of calculated positions over the footprint of the plant - configuration $\mathrm{f}$ ): 4 modules, filter on

\section{CONCLUSION AND FUTURE WORK}

When RSSI values are used as input parameters of the system additional measures are necessary to increase the availability and the accuracy of the localization system. The presented results show that an increased accuracy of the location estimation can be achieved with the chosen diversity concept and plausibility filtering. Thus it is possible to generate a rough movement pattern of an unknown node also with low infrastructural costs. The localization is more robust against signal fading and frequency specific interferer.

As an outlook for further developments we consider to use a triaxial acceleration sensor on the BN to reach a better understanding of the system's dynamics. With the acceleration information it might be possible to assign special variations in RSSI values either to a real movement of the mobile device or to signal fading. With the extension of the sensor and a motion-triggered position request also the operating time of the battery-powered $\mathrm{BN}$ could be extended significantly.

\section{ACKNOWLEDGEMENTS}

The represented work was supported by the program "Furderung von Innovativen Netzwerken (InnoNet)" of the Federal Ministry of Economics and Technology (BMWi) under the subsidy indicators 16IN0428 and 16IN0429.

\section{REFERENCES}

[1] H. Beikirch, M. Voß, S. Ganzel, and A. Fink. "Erhöhung funktionaler Sicherheit bei drahtloser Kommunikation in industriellen Anwendungen". In 5th International Symposium on Automatic Control, 2008.

[2] J. Hightower and G. Boriello. A Survey and Taxonomy of Location Systems for Ubiquitous Computing. Technical report, University of Washington, Department of Computer Science and Engineering, 2001.

[3] T. Benkner. Grundlagen des Mobilfunks. Schlembach Verlag, 2007.

[4] A. Fink. Sicherheitsrelevante Funkkommunikation mit Positionsbestimmungsunterstützung. Master's thesis, Universität Rostock, 2008.

[5] A. Fink, H. Beikirch, and M. Voß. "Radio Communication and Localization in Functional Safety Applications of Industrial Automation". In Embedded World 2009, Proceedings, Ses. 1.2-5, pages 1-6. WEKA Fachmedien $\mathrm{GmbH}$, 2009.

[6] H. Beikirch, M. Voß and A. Fink. "Redundancy Approach to Increase the Availability and Reliability of Radio Communication in Industrial Automation". In IEEE Conference on Emerging Technologies \& Factory Automation (ETFA), 2009.

[7] M. Rehim. Horus: A WLAN-based Indoor Location Determination System. PhD thesis, University of Maryland, 2004.

[8] T. S. Rappaport. Wireless Communications Principles and Practice. Prentice Hall PTR, 2002. 
[9] R. Behnke and D. Timmermann. "AWCL: Adaptive Weighted Centroid Localization as an Efficient Improvement of Coarse Grained Localization". In 5th Workshop on Positioning Navigation and Communication, pages 243250, 2008.

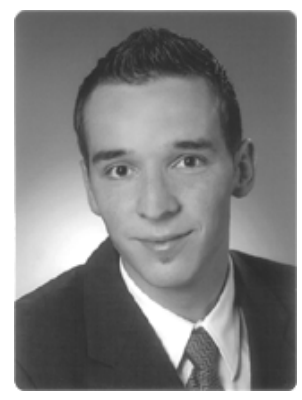

Andreas Fink, received his M.Sc. degree in information technology from the University of Rostock in 2008. Currently he works as a research scientist at the Department of Computer Science and Electrical Engineering.

His primary research interests are powerline communication interfaces, wireless communication systems and indoor localization.
Helmut Beikirch, to hold since 1998 the professorship "Electronic Devices and Circuit Design" of the Faculty of Computer Science and Electrical Engineering at the University of Rostock. $\mathrm{He}$ is the Head of the research group in electronic circuit design and communication systems in industrial automation.

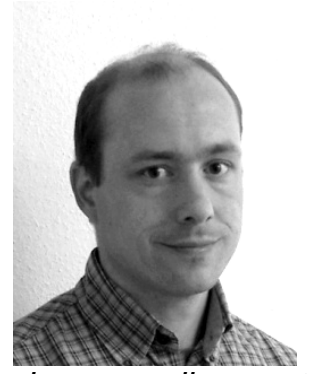

Matthias Voß, received his Dr.-Ing. degree from University of Rostock in 2003. Now he belongs to the scientific staff of the Faculty of Computer Science and Electrical Engineering at the University of Rostock.

His area of interests includes powerline systems and wireless communication systems in the field of industrial automation. 